\title{
Frequency Dependence of Fading Statistics for Ultrawideband Systems
}

\author{
Wasim Q. Malik, Member, IEEE, David J. Edwards, and Christopher J. Stevens
}

\begin{abstract}
The variation of channel fading and dispersion statistics between the ultrawideband (UWB) channel subbands is characterized experimentally. Within the FCC-allocated UWB frequency range $(3.1-10.6 \mathrm{GHz})$, the multipath characteristics in various subbands are found to be highly dissimilar. It is demonstrated that the higher subbands suffer greater attenuation, and the rms delay spread varies significantly with the center frequency. The impact of this variation on multiband-OFDM UWB system performance is evaluated, and a large performance variation across the band is reported.
\end{abstract}

Index Terms - Channel characterization, frequency-selective, multiband OFDM (MB-OFDM), ultrawideband (UWB).

\section{INTRODUCTION}

$\mathbf{U}$ LTRAWIDEBAND (UWB) technology is a key contender for a range of wireless applications such as multimedia communications, sensor localization, radars and imaging systems [1]. The design of a reliable UWB communications system depends on the accurate characterization of the propagation channel, which determines the limits on the achievable throughput and coverage range. Several UWB propagation studies have been reported recently (see [2] for a good overview). These studies, however, treat the entire frequency band as a whole, providing little insight into the frequency-dependent effects at the subband level. The consideration can be significant, as the operating frequency influences transmission through objects, diffraction and scattering [3], [4]. System components such as antennas, amplifiers, and filters also contribute to the link frequency selectivity [5]. At the receiver, these effects jointly influence the multipath profile, and in turn the channel fading statistics such as pathloss and delay spread, creating the possibility of some performance variation between subbands. Increasing interest in multiband UWB technologies, such as multiband orthogonal frequency division multiplexing (MB-OFDM), soft-spectrum adaptation, and orthogonal pulse waveform design, further presents the need for frequency-dependent propagation analysis. This information can be used to adapt a system to the environment for optimal performance by pre- or post-compensation using flexible spectral control techniques such as cognitive radio.

Based on this motivation, this work evaluates the dependence of the channel statistics on the operating center frequency within the UWB band. The measured UWB signal spectrum is partitioned into a number of subbands, and the

Manuscript received July 21, 2005; revised March 9, 2006; accepted July 6 , 2006. The associate editor coordinating the review of this letter and approving it for publication was Z. Tian. This work was supported in part by the UK Engineering and Physical Sciences Research Council via Grant GR/T21769/01.

The authors are with the Department of Engineering Science, University of Oxford, Parks Road, Oxford OX1 3PJ, UK (email: wasim.malik@eng.ox.ac.uk).

Digital Object Identifier 10.1109/TWC.2007.05490. corresponding pathloss variation and dispersion is characterized statistically. The impact of frequency-dependent channel statistics on system design and performance is illustrated with MB-OFDM system simulation results obtained using measured channel responses. The scope of this letter is restricted to highlighting the impact of a shortcoming in existing approaches to UWB channel characterization and system modeling. Detailed subband-level channel characterization based on extensive measurement campaigns is therefore left as a future exercise. The next section describes the procedure for conducting the channel measurements used in this work.

\section{EXPERIMENTAL CONFIGURATION}

Coherent channel sounding was performed using a vector network analyzer (VNA) in an indoor office environment. The FCC-approved $7.5 \mathrm{GHz}$ wide frequency range between $f_{l}=3.1 \mathrm{GHz}$ and $f_{h}=10.6 \mathrm{GHz}$ was sounded at $n_{f}=1601$ discrete, uniformly-spaced frequency points, with frequency resolution $\Delta f=\left(f_{h}-f_{l}\right) /\left(n_{f}-1\right) \approx 4.7 \mathrm{MHz}$. The equipment, including the VNA, cables, connectors and a lownoise amplifier, was calibrated to remove frequency-dependent attenuation and phase distortion prior to the measurement. Discone antennas, with omni-directional radiation patterns, were used at both ends (for details on the construction of the antennas, see [5]). The antennas were vertically polarized and positioned at a height of $1.5 \mathrm{~m}$ above the ground.

The propagation environment consisted of a $6 \mathrm{~m} \times 6 \mathrm{~m}$ room with block walls, glass windows and several metallic objects of various sizes, providing a rich scattering environment. Line-of-sight (LOS) and obstructed-line-of-sight (OLOS) scenarios were measured within the room; the blockage for the latter was created by placing a large, grounded aluminum sheet in the direct propagation path. The receiving antenna was mounted on a computer-controlled positioning arm and translated on a horizontal grid over $1 \mathrm{~m}^{2}$ to measure the local variation. The transmitting antenna was fixed at a location $4.5 \mathrm{~m}$ away from the center of the grid. The channel measurement was conducted at 100 positions along each dimension of the receiving grid, with individual positions $\Delta x=0.01 \mathrm{~m}$ apart in each direction, yielding $n_{x}=10000$ channel responses for each propagation scenario.

\section{Channel Data Analysis}

The complex channel transfer function (CTF) measured at the $x^{\text {th }}$ receiver location can be expressed mathematically as

$$
H_{x}(f)=\sum_{k=1}^{n_{f}} A_{x, k} e^{j \psi_{x, k}} \delta(f-k \Delta f), \quad f_{l} \leq f \leq f_{h},
$$

where $A_{x, k}$ and $\psi_{x, k}$ are the magnitude and phase responses at the $k^{\text {th }}$ frequency component. 
The complex impulse response (CIR) of the channel is obtained from $H_{x}(f)$ using the inverse discrete Fourier transform. Based on the measurement dynamic range and inspection of the received signals, the noise threshold is set at $25 \mathrm{~dB}$ below the strongest multipath component (MPC) in order to remove the noise and residual signal from further processing. The resulting CIR, after the removal of the propagation delay, is given by

$$
h_{x}(\tau)=\sum_{n=0}^{N-1} a_{x, n} e^{j \phi_{x, n}} \delta(\tau-n \Delta \tau),
$$

where $a_{x, n}$ and $\phi_{x, n}$ are the magnitude and phase responses in the $n^{\text {th }}$ time-bin, $\tau$ represents the time delay with respect to the direct path, $\Delta \tau=\left(f_{h}-f_{l}\right)^{-1}$ is the time-resolution, $N=\tau_{\max } / \Delta \tau$ is the number of discrete time-bins in the CIR, and $\tau_{\max }$ is the maximum excess delay defined in Sec. IV. To extract the multipath gain and delay parameters characterized in the subsequent analysis, the resolvable MPCs are identified from the instantaneous power delay profile (PDP),

$$
p_{x}(\tau)=\left|h_{x}(\tau)\right|^{2}=\sum_{n=0}^{N-1} a_{x, n}^{2} \delta(\tau-n \Delta \tau),
$$

through local maxima detection.

For the investigation of frequency-dependent small-scale fade variation, the UWB spectrum is divided into subbands $b=\{1, \ldots, 5\}$, each $1.5 \mathrm{GHz}$ wide. The subbands, each of length $\left(n_{f}-1\right) / 5$ now, are zero-padded to restore the length for fair comparison. If $f_{b, l}$ and $f_{b, h}$ refer to the low- and highfrequency boundaries of subband $b$, its transfer function is

$$
H_{x, b}(f)= \begin{cases}H_{x}(f), & \text { if } f_{b, l} \leq f \leq f_{b, h} \\ 0, & \text { otherwise. }\end{cases}
$$

Note that the discrete representation of $H_{x, b}(f)$ now also contains $n_{f}$ points and $\Delta \tau$ is maintained. The subband CIR,

$$
h_{x, b}(\tau)=\sum_{n=0}^{N-1} \alpha_{x, b, n} e^{j \phi_{x, b, n}} \delta(\tau-n \Delta \tau),
$$

is calculated using the Fourier relation, where $\alpha_{x, b, n}$ and $\phi_{x, b, n}$ are the magnitude and phase responses at the respective frequency components in the $b^{\text {th }}$ subband.

As a representative example, Fig. 1 shows the subband CIRs for the measured LOS channel at one location. The CIRs are remarkably dissimilar, as evident from inspection, in terms of the multipath amplitudes and delays, establishing the strong dependence of the channel response on the frequency subband. It also illustrates that not all MPCs contain the full frequency content, a situation that can affect UWB link performance significantly. With this preliminary evidence, the next section quantifies the dependence of the channel response on the center frequency in terms of the fading statistics.

\section{Channel Statistics}

For each measurement point, the subband fading statistics are derived using the absolute noise level of the corresponding full-band CIR as the threshold. The characterization of the subband pathloss, delay spread and multipath, using their first order statistics, is undertaken in this section.

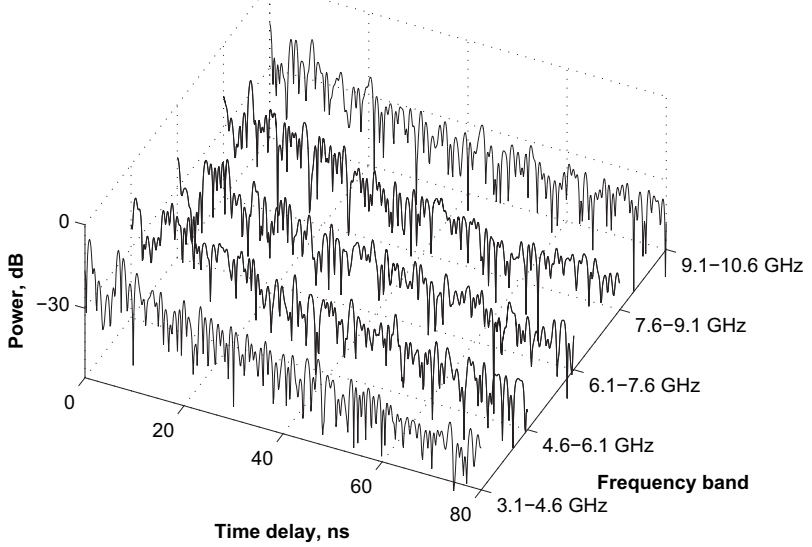

Fig. 1. The complex impulse responses of (CIRs) for the LOS UWB channel subbands obtained from a single measured full-band channel transfer function. The time delay is with reference to the first arrival in the full-band CIR, and the powers are normalized to the full-band CIR's peak power.

\section{A. Pathloss Variation}

The pathloss determines the mean received power and thus affects the link budget and link quality. Accurate pathloss characterization is especially important for power-limited UWB systems that typically operate at low SNR. The pathloss dependence on frequency $f$ arises primarily from the antenna power density and gain variation with $f$ [5]. Additionally, frequencyselective physical propagation phenomena such as diffraction, scattering and material properties can cause frequencydependent pathloss [2], [6]. In a frequency-selective channel, pathloss can be expressed using the general form

$$
\begin{aligned}
P L_{[\mathrm{dB}]}(f, d)= & P L_{[\mathrm{dB}]}\left(f_{0}, d_{0}\right)+10 n \log _{10}\left(\frac{d}{d_{0}}\right) \\
& +10 m \log _{10}\left(\frac{f}{f_{0}}\right)
\end{aligned}
$$

where $d_{0}$ and $f_{0}$ represent the reference distance and frequency, while $n$ and $m$ denote the distance- and frequencydependent pathloss exponents, respectively. The above relation indicates that the subbands within the UWB frequency range will experience dissimilar pathloss if $m \neq 0$. In a given environment, $m=2+\sigma_{m}$ for omni-directional antennas, where $\sigma_{m}$ is a random variable representing a small offset and spread in $m$ due to propagation effects [6].

To investigate the frequency-dependent pathloss further, the wideband energy of subband $b$ at location $x$ can be found as

$$
P_{x, b}=\int_{f_{b, l}}^{f_{b, h}}\left|H_{x}(f)\right|^{2} \mathrm{~d} f=\int_{-\infty}^{\infty}\left|H_{x, b}(f)\right|^{2} \mathrm{~d} f .
$$

The signal energy calculation is actually performed in time domain after thresholding in order to reduce the effect of noise. Using the Parseval relation, the subband energy, $P_{x, b}$, can be evaluated from the subband PDP, $p_{x, b}(\tau)$, by the integration of the multipath energy over the time-dispersed received symbol duration, $\tau_{\max }$. Assuming an optimal receiver, such as an $L_{b^{-}}$ finger maximal-ratio combining rake, and using our discrete 
treatment from (1), we can write

$$
P_{x, b}=\sum_{\tau=0}^{\tau_{s}} p_{x, b}(\tau)=\sum_{l=1}^{L_{b}} p_{x, b}(l),
$$

where $L_{b}$ is the number of MPCs in $b$, and $p_{x, b}(l)$ represents the energy in the $l^{\text {th }}$ MPC. The energy in each subband is normalized with respect to the full-band energy, $P_{x}$, at that location, to obtain a reference pathloss figure, so that the normalized subband energy, representing the subband pathloss, becomes $P_{x, b}^{\prime}=P_{x, b} / P_{x}$, where $P_{x}=\sum_{l=1}^{L} p_{x}(l)$.

Statistical analysis is performed next to obtain the distribution of $P_{x, b}^{\prime}$ in LOS and OLOS scenarios. From a comparison of the cumulative distribution functions (CDFs) in Fig. 2, a difference of up to $10 \mathrm{~dB}$ is observed between the mean pathloss of the highest and lowest subbands, increasing monotonically with frequency. Table I shows that the pathloss standard deviation also increases in the higher bands. The mean pathloss of the mid-band range is about $14 \mathrm{~dB}$ higher than the full-band pathloss. This observation indicates the inaccuracy introduced by approximating the pathloss of the whole band with that of the center frequency, $f_{c}$, as assumed in simple channel models. It emphasizes that the individual pathloss of each subband must be taken into account during system design. This characterization thus quantifies the observations of Fig. 1, where a large power variation for the dominant MPCs across the subbands is clearly noticeable.

\section{B. Time Dispersion}

The time dispersion of a channel can be characterized in terms of the delay spread, which depends on the location and composition of scatterers and the overall geometry of the propagation environment. It provides information about the extent of frequency-selective fading, and limits the highest achievable data-rate. The delay parameters are extracted directly from the PDP. We will analyze three of the delay parameters for each subband, namely the mean excess delay, rms delay spread, and maximum excess delay [7]. The mean excess delay is defined as the first central moment of the PDP, i.e.,

$$
\tau_{m}=\sum_{n=0}^{N-1} \alpha_{n}^{2} \tau_{n}\left(\sum_{n=0}^{N-1} \alpha_{n}^{2}\right)^{-1}
$$

where $\tau_{n}$ denotes the delay of the $n^{\text {th }}$ MPC. The rms delay spread is the second central moment of the PDP given by

$$
\tau_{r m s}=\sqrt{\overline{\tau^{2}}-\tau_{m}^{2}}
$$

The maximum excess delay, $\tau_{\max }$, is the delay of the last resolved MPC above the noise threshold, i.e.,

$$
\tau_{\max }=\sup _{n}\left\{\tau_{n}\right\}
$$

From Fig. 3, there is a variation of 3-4 ns in $\tau_{r m s}$ between the subbands, while the full-band $\tau_{r m s}$ is consistently larger than the subband $\tau_{r m s}$. Table I shows that the subband $\tau_{m}$ varies on average by 3-5 ns. The difference in the subband $\tau_{\max }$ is $20-25 \mathrm{~ns}$. In the lowest subband, $\tau_{\max }$ is $10-15 \mathrm{~ns}$ smaller than that in the full-band, and the remaining subbands follow. The smaller delay spread observed in the higher

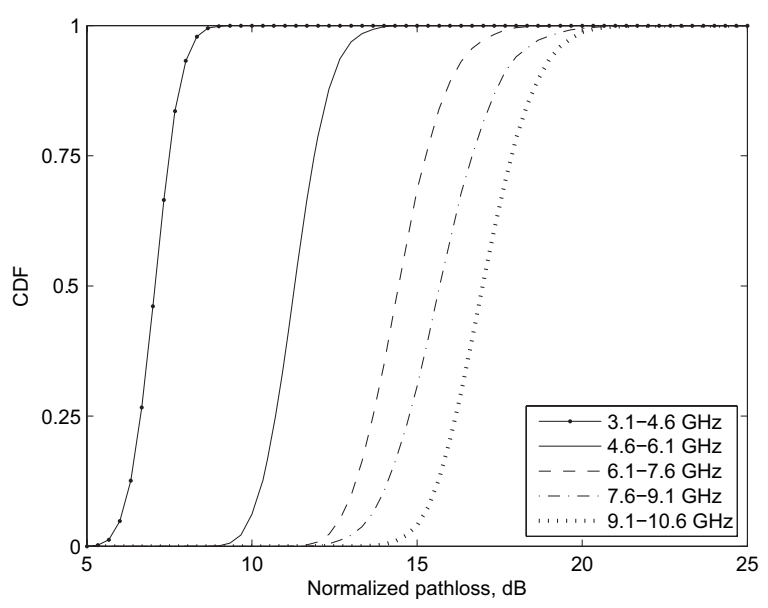

(a)

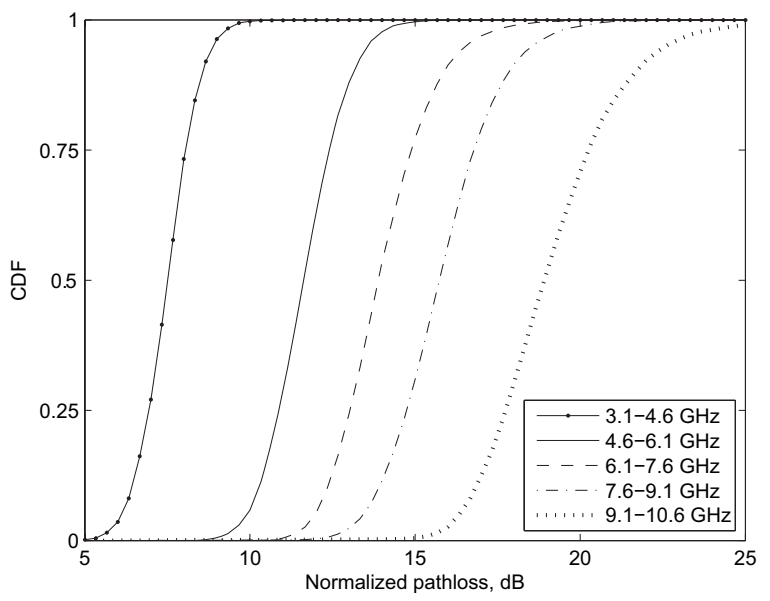

(b)

Fig. 2. Cumulative distribution functions (CDFs) of subband pathloss normalized to the full-band pathloss in (a) LOS and (b) OLOS channels.

subbands is due to greater attenuation, causing the MPCs to rapidly fall below the PDP threshold. From our measurements, the variation of these statistics with the center frequency does not, however, appear to be monotonic. Some of the OLOS PDPs in the highest subband carry no signal energy, indicated by a finite probability of the event $\tau_{\max }=0$ in Table I. From this analysis, we find that the full-band UWB channel cannot be treated homogeneously for dispersion characterization.

\section{Number of Multipath Components}

Multipath resolution is an important factor in the small-scale fading behavior of a channel, as the high multipath resolution of wideband systems leads to reduced fade depths. The system bandwidth directly affects the time resolution and thus the number, $L$, of individually resolved MPCs. It is important, therefore, to reiterate that in our analysis, the subbands have equal bandwidth and differ only in their center frequencies. Table I lists the first order statistics of the number of MPCs, $L_{b}$, for each subband, and also of $L$ for the full band. The latter is almost five times greater, on average, due to the five-fold bandwidth of the full-band channel. The subbands, however, have progressively decreasing $L_{b}$ with frequency, due to dropping power levels and frequency-selective fading as 
TABLE I

THE MEAN FADING AND DISPERSION STATISTICS FOR MEASURED UWB CHANNELS, WITH THE STANDARD DEVIATION SHOWN IN PARENTHESES

\begin{tabular}{|c|c|c|c|c|c|c|c|c|c|c|}
\hline \multirow{2}{*}{ Band, GHz } & \multicolumn{2}{|c|}{ Normalized Pathloss, dB } & \multicolumn{2}{|c|}{ Rms Delay Spread, ns } & \multicolumn{2}{|c|}{ Mean Excess Delay, ns } & \multicolumn{2}{|c|}{ Max. Excess Delay, ns } & \multicolumn{2}{|c|}{ Num. of MPCs } \\
\hline & LOS & OLOS & LOS & OLOS & LOS & OLOS & LOS & OLOS & LOS & OLOS \\
\hline $3.1-10.6$ & $0(0)$ & $0(0)$ & $7.8(0.9)$ & $7.7(1.2)$ & $8.2(0.9)$ & $10.0(1.7)$ & $50.6(6.7)$ & $50.9(8.2)$ & $90.1(11.8)$ & $89.8(16.7)$ \\
\hline $3.1-4.6$ & $7.2(0.6)$ & $7.7(0.8)$ & $7.0(1.0)$ & $6.6(1.3)$ & $8.3(1.0)$ & $8.7(1.7)$ & $37.8(5.8)$ & $35.8(7.0)$ & $30.1(4.9)$ & $26.7(6.4)$ \\
\hline $4.6-6.1$ & $11.5(0.9)$ & $11.8(1.1)$ & $6.4(1.0)$ & $5.9(1.4)$ & $8.4(1.2)$ & $9.3(1.7)$ & $29.4(6.3)$ & $28.7(7.6)$ & $20.7(4.3)$ & $18.8(5.6)$ \\
\hline $6.1-7.6$ & $14.7(1.2)$ & $14.2(1.4)$ & $4.0(0.8)$ & $3.8(1.2)$ & $7.8(0.7)$ & $5.5(2.5)$ & $19.5(4.7)$ & $17.4(7.1)$ & $12.1(3.1)$ & $11.8(4.2)$ \\
\hline 7.6-9.1 & $15.9(1.4)$ & $16.0(1.6)$ & $3.9(1.0)$ & $3.4(1.4)$ & $6.9(0.9)$ & $3.6(2.2)$ & $16.9(4.2)$ & $13.1(6.2)$ & $9.6(3.0)$ & $8.3(4.2)$ \\
\hline $9.1-10.6$ & $17.2(1.2)$ & $19.3(2.8)$ & $4.8(1.0)$ & $3.5(1.6)$ & $5.5(1.2)$ & $3.7(2.4)$ & $16.2(5.1)$ & $11.4(6.9)$ & $8.6(3.1)$ & $6.5(4.5)$ \\
\hline
\end{tabular}

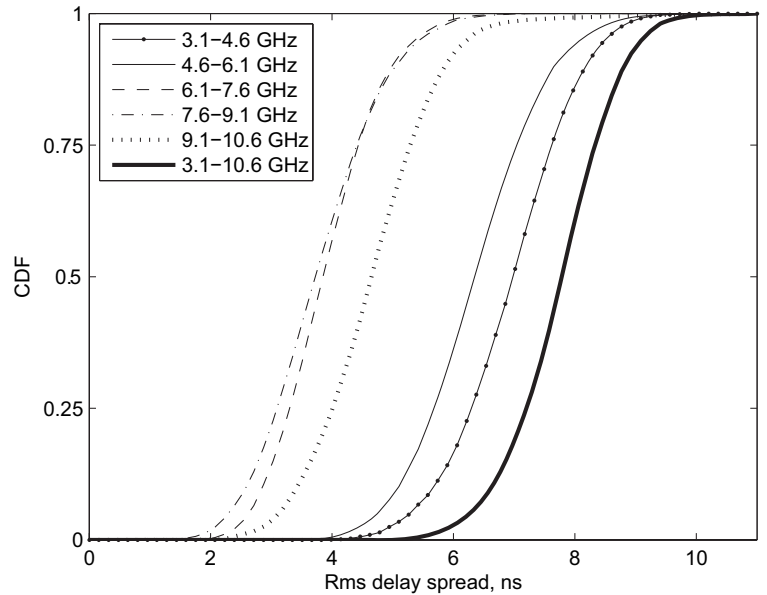

(a)

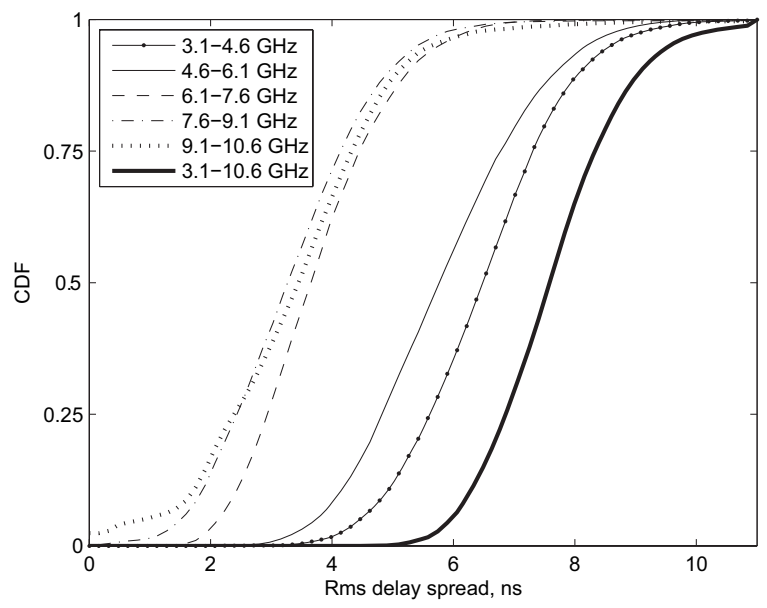

(b)

Fig. 3. Cumulative distribution functions (CDFs) of the rms delay spread in the subbands in (a) LOS and (b) OLOS channels.

suggested earlier by Fig. 1 . The range of $L_{b}$ across the UWB band indicates that the lowest subband receives 20 additional components as compared to the highest subband. In this measurement dataset, the OLOS channel experiences a slightly smaller $L$ than the LOS channel because of the blockage of the signal cluster within a small angular spread of the direct path, while the specular components are unaffected. The standard deviation of $L$ for OLOS is, however, higher because of greater distribution of energy among the MPCs and the consequently larger variation in their gains and detectability.

\section{ImpaCt ON SYSTEM PERformance}

A single-band pulsed UWB architecture, such as the impulse radio [8], uses carefully constructed signal waveforms and correlation receivers. It can be shown that power and pathloss variation with frequency can lead to spectral distortion and thus a modification of the waveform shape during transmission, degrading the correlation receiver output [6]. Furthermore, the dissimilar spectra of the MPCs result in individual waveform distortion for each, lowering its correlation with the template waveform at the receiver in an unpredictable manner in the absence of suitable channel compensation on a per-path basis [4]. In addition, the higher pathloss observed in the higher frequency regions of the signal spectrum reduces the effective operating bandwidth for the UWB system, which can thus reduce its frequency diversity, increase the outage probability, and lower the achievable capacity. Similarly, the variation of delay spread with frequency can affect the bit error rate (BER) and achievable capacity within the subbands due to the varying levels of inter-symbol interference (ISI).

We now evaluate the bit-error rates (BERs) obtained in the individual UWB subbands with Monte-Carlo simulations of an MB-OFDM system using the measured short-range LOS channels. We consider a 53.3 Mbps implementation based on the specification in [9], and therefore redefine our subbands to be $528 \mathrm{MHz}$ wide, with the subband spectra extracted once again from our measured full-band channel data. The system includes a 1/3-rate convolutional encoder and Viterbi decoder for forward error correction, and a pertone zero-forcing equalizer. Digital baseband data modulation is performed using quadrature phase shift keying (QPSK), and OFDM is implemented using the fast Fourier transform (FFT) with 128 subcarriers spaced $4.125 \mathrm{MHz}$ apart. A zero-pad (ZP) sequence of 32 samples and $60.6 \mathrm{~ns}$ duration is appended to each OFDM symbol in order to capture the multipath and mitigate the ISI. In contrast with [9], subband switching within the bandgroups is disabled so that the individual subband performances can be compared.

Fig. 4 shows the BER as a function of the transmit signal bit energy to noise ratio when the transmission is in the specified subband. For presentation clarity, we show only a representative subset of the available 14 subbands. Despite the fact that the ZP duration is longer than the typical maximum excess delay spread in the propagation scenario considered, the BER is found to vary considerably between the subbands. From the figure, the difference in the required $E_{b} / N_{0}$ at a BER of $10^{-3}$ for subbands 3 and 14 is $7 \mathrm{~dB}$, and increases further 


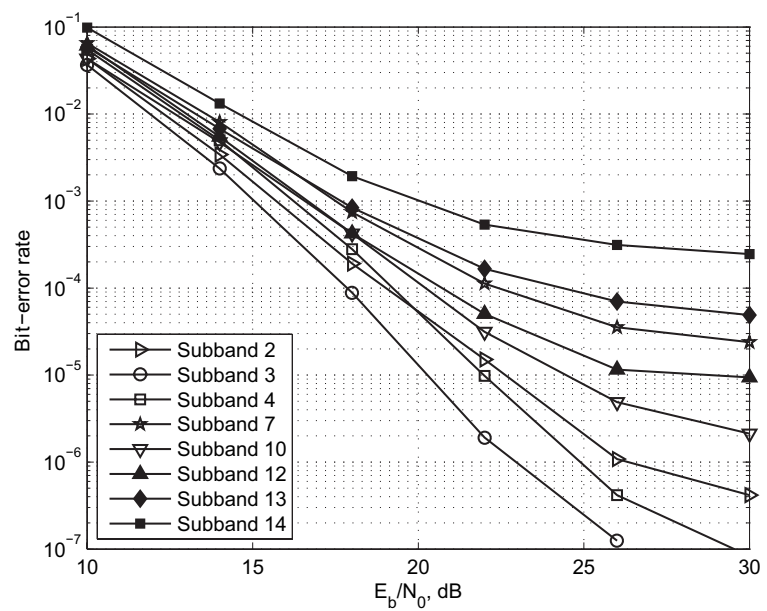

Fig. 4. Some representative bit-error rate (BER) curves for the multibandOFDM subbands using measured channels.

when a lower value of the BER is considered. The curves for the other subbands shown in the figure lie in between. Thus a performance difference of up to three orders of magnitude between the subbands is in evidence.

From our results, although in general the BER tends to increase in the higher subbands, the center frequency does not appear to completely dictate the BER behavior. At first glance, the $E_{b} / N_{0}$ difference may simplistically appear to be dependent on the pathloss variation between the subbands (see Table I). Detailed analysis, however, shows that the BER is not strictly monotonic with respect to the center frequency, similar to the delay spread, suggesting that the monotonically increasing frequency-dependent pathloss does not completely account for this variation. The MB-OFDM performance can thus be conjectured to be a joint function of the pathloss, multipath, noise, and interference effects.

\section{CONCLUSION}

The characterization of the indoor UWB propagation channel in this letter has shown that the fading statistics are nonuniform over the channel bandwidth. Strong dependence of the pathloss and delay spread on the center frequency has been reported. It has been shown that the higher frequency components suffer greater attenuation, with up to $10 \mathrm{~dB}$ of pathloss difference between the extreme subbands. The delay spread analysis shows that the subbands experience varying levels of dispersion. The number and gains of the resolved MPCs also vary between the subbands, suggesting that some of the MPCs span only a certain portion of the signal bandwidth. Furthermore, our error-rate simulations of multiband UWB systems using measured channels have shown that the subband $E_{b} / N_{0}$ can vary by up to $7 \mathrm{~dB}$. In view of these findings, it can be concluded that the conventional spectrally unified description of UWB channel fading statistics can be misleading, and subband-level channel modeling and system analysis is vital for robust design.

The results also suggest the possibility of a certain performance improvement achievable by using only a subset of the available subbands in a closed-loop scheme. In contrast with the current MB-OFDM implementation [9] that operates only over the first bandgroup comprising the first three subbands, a subband-selection scheme based on subband channel fading characteristics is likely to provide improved performance. Suggested future work can therefore consider adaptive UWB transmission schemes catering to subband-specific channel characteristics.

\section{ACKNOWLEDGMENT}

The authors would like to thank Nyembezi Nyirongo for assisting with MB-OFDM simulations.

\section{REFERENCES}

[1] B. Allen, M. Döhler, E. E. Okon, W. Q. Malik, A. K. Brown, and D. J. Edwards, Eds., Ultra-Wideband Antennas and Propagation for Communications, Radar and Imaging. Wiley, 2006.

[2] A. F. Molisch, "Ultrawideband propagation channels-theory, measurement, and modeling," IEEE Trans. Veh. Technol., vol. 54, no. 5, Sept. 2005.

[3] K. Heidary, "Ultra-wideband (UWB) incidence on multiple dielectric interfaces," in Proc. IEEE Ant. Propagat. Soc. Int. Symp. (APS) 2004.

[4] R. C. Qiu, "A generalized time domain multipath channel and its applications in ultra-wideband (UWB) wireless optimal receiver designpart II: physics-based system design," IEEE Trans. Wireless Commun., vol. 3, no. 6, Nov. 2004.

[5] W. Q. Malik, D. J. Edwards, and C. J. Stevens, "Angular-spectral antenna effects in ultra-wideband communications links," IEE Proc. Commun., vol. 153, no. 1, Feb. 2006.

[6] _ "Frequency-dependent pathloss in indoor ultrawideband channels," in Proc. IEEE Int. Conf. Commun. (ICC) 2006.

[7] T. S. Rappaport, Wireless Communications: Principles and Practice. Prentice Hall, 2001.

[8] M. Z. Win and R. A. Scholtz, "Impulse radio: how it works," IEEE Commun. Lett., vol. 2, no. 2, Feb. 1998.

[9] A. Batra, et al., "Multiband OFDM physical layer proposal for IEEE 802.15 Task Group 3a," IEEE, Tech. Rep. P802.15-03/268r0-TG3a, July 2003. 